Mustafa Calis and Marc P.Y. Desmulliez

\title{
Haptic sensing technologies for a novel design methodology in micro/nanotechnology
}

ABSTRACT. This paper presents a new design methodology for micro/nanotechnology based on haptic sensing technologies. The methodology enables designers to model and interact in real time with their virtual prototype. One of the main advantages of haptic sensing is the ability to bring the most important microscopic forces back to the designer's world. Other significant benefits for developing such a methodology include gain productivity and the capability to include manufacturing costs within the design cycle.

Nanotechnology Perceptions 1 (2005) 89-97

Nonsubscribers: purchase individual article 